\title{
A TDMA-Based MAC Protocol with Cooperative Diversity
}

\author{
Zhuo Yang, Yu-Dong Yao, Xiaochen Li, and Di Zheng
}

\begin{abstract}
Cooperative diversity is an effective technique for transmission performance improvement over fading channels. In this paper, through the use of a virtual antenna array, a time division multiple access (TDMA) based cooperative media access control (MAC) protocol, Cooperative TDMA (C-TDMA), is proposed to enable cooperative transmissions in Rayleigh fading channels to improve the probability of correct packet reception and system throughput. A C-TDMA throughput expression is derived using a Markov chain model. Numerical results show that the throughput of conventional TDMA can be improved by over $40 \%$ through the use of the cooperative MAC designs.
\end{abstract}

Index Terms-Cooperative diversity, media access protocol, throughput, TDMA.

\section{INTRODUCTION}

$\mathbf{C}$ OOPERATIVE communication is a very attractive research topic for improving the effective quality of service in wireless networks. Appropriate medium access strategies are required in order to exploit the physical layer advantages provided by cooperative communications [1]. In [2] and [3], medium access control (MAC) protocols enabling cooperation in 802.11 systems are considered. In [4], Cooperative Aloha (C-Aloha) is proposed to enable cooperative transmissions based on multiple-antenna systems for packet retransmissions to obtain the transmit diversity. According to numerical results, this cooperative MAC design [4] introduces a 30\% increase in the maximum throughput of slotted Aloha systems. Furthermore, C-Aloha can be applied to other contention based MAC protocols.

A cooperative MAC design for TDMA systems has not been explored thoroughly. In [5], a fixed relay/wireless router utilizes the idle time slots available in a TDMA frame and two corresponding MAC protocols are designed to assist packet retransmissions. Although this method improves packet retransmissions when idle time slots are available, it becomes less effective when the traffic load is high (few idle slots are available). In this paper, we introduce a Cooperative TDMA (CTDMA) protocol to enable cooperative transmissions through virtual antenna arrays [6] to obtain diversity gain. In C-TDMA, each terminal has two tasks. First, a terminal needs transmits its own data packet during its allocated time slot in each frame. Second, it monitors other time slots in each frame and cooperates with other terminals to retransmit failed packets due to channel impairments during previous frames. In other words, neighbor terminals will help a source packet terminal to retransmit its lost packets during the time slot of the

Manuscript received December 21, 2009. The associate editor coordinating the review of this letter and approving it for publication was A. Banchs.

The authors are with the Dept. of Electrical and Computer Engineering, Stevens Institute of Technology, Hoboken, NJ 07030 (e-mail: \{zyang4, yudong.yao,dzheng\}@stevens.edu).

Digital Object Identifier 10.1109/LCOMM.2010.06.092451 original source packet terminal. This cooperative MAC design effectively overcomes severe channel impairments because of fading, increases the probability of correct packet reception, and thus results in throughput improvements. C-TDMA can also be extended to other control or schedule based MAC protocols to obtain transmit diversity .

The rest of this paper is organized as follows. Section II describes C-TDMA in details. Section III derives the throughput of the conventional TDMA and C-TDMA system in Rayleigh fading channels. Analytical and simulation results are given in Section IV and, finally, Section V draws conclusions.

\section{Cooperative TDMA}

In cooperative TDMA uplink scheduling for $N$ mobile terminals and a base station, a frame contains $N$ time slots with equal time length. Each time slot is exclusively allocated to a terminal. All terminals are assumed to be synchronized and all packets have equal length. Moreover, we assume that packet arrivals at each terminal are independent and follow a Bernoulli process [5] [7]. Specifically, each terminal generates a new packet with probability $\sigma$ during each time slot, and the probability of no packet generation is $1-\sigma$. Each C-TDMA terminal has a first-in and first-serve (FIFS) buffer of length $L$, and a parallel output buffer of length $N-1$ for cooperative transmissions. Finally, we assume that immediate feedback (positive or negative acknowledgement) from the base station at the end of each time slot can be received by each terminal through an error-free channel [4].

In a cooperative TDMA system, each terminal transmits existing packets, if any, in its FIFS buffer during its exclusively allocated time slot. If the transmission fails, the packet will be retransmitted in the following frames until it is transmitted successfully and then cleared from the terminals' FIFS buffer. During other slots other than the terminal's exclusively allocated slot within a frame, called overhearing slots, each terminal listens to other terminals' transmissions. At the end of each overhearing slot, there are four possible scenarios: a) the packet overheard is correctly decoded and a positive acknowledgement is received by the terminal; b) the packet overheard is not correctly decoded and a positive acknowledgement is received by the terminal; c) the packet overheard is correctly decoded but a negative acknowledgement is received by the terminal; d) the packet overheard is not correctly decoded and a negative acknowledgement is received by the terminal. For scenario c), the terminal will load the successfully decoded (overheard) packet into its corresponding parallel output buffer. For scenarios a), b) and d), the overheard packet will be discarded. In the following frames, this terminal with the successfully decoded packet will serve as a cooperative terminal with probability $q_{c}$, and 
transmit this packet together with the original source terminal during the source terminal's exclusively allocated time slot and thus result in diversity gain.

Considering $k-1$ cooperative terminals plus the source terminal, it forms a $k$-element virtual antenna array (e.g., in the form of multiple-input single-output (MISO)). The performance of this antenna array can be evaluated following that of a maximal-ratio-combing (MRC) receiver [8]. The improvement of the successful packet reception probability depends on the number of cooperating terminals which determines the number of branches in MRC. Assuming a binary phase shift keying (BPSK) modulation scheme, the average bit error rate (BER), $\bar{P}_{b}$, in a Rayleigh fading channel for a MRC receiver with $k$-branch diversity is given by [4] [9] $\bar{P}_{b}(\bar{\gamma}, k)=\frac{1}{\pi} \int_{0}^{\pi / 2}\left(1+\frac{\bar{\gamma}}{\sin \phi^{2}}\right)^{-k} d \phi$, where $\bar{\gamma}$ is the average signal noise ratio (SNR) of each link. Without considering channel coding, the success probability of a $\mu$ bit length packet can be calculated by $P_{s}(k)=\left[1-\bar{P}_{b}(\bar{\gamma}, k)\right]^{\mu}$ and thus the packet error rate is $P_{e}(k)=1-P_{s}(k)$. Here, we assume that SNR can be estimated at the base station and is static during one time slot.

\section{Throughrut AnAlysis of C-TDMA}

In this section, we develop analytical models for conventional TDMA and C-TDMA systems in terms of network throughput in Rayleigh fading channels. In order to derive the throughput of conventional TDMA/C-TDMA, we model the system as a Markov chain [7] [10]. For this constructed Markov chain, a state $U_{i}$ is defined to describe $i$ packets in the length $L$ FIFS buffer of a terminal. Setting the observation point at the end of the terminal's transmission slot in each frame, we have $0 \leq i \leq L$ states. In this paper, we only consider the case $L \leq N$. Next step, we resolve the Markov chain after obtaining its one step transition matrix. Finally, the throughput of conventional TDMA/C-TDMA can be obtained.

We first consider the Markov chain of the conventional TDMA system. Its one step transition matrix $\left\{B_{T}\right\}_{i, j}$ can be calculated following the method introduced in [7]. This one step transition matrix is given in Eq. (1), where $P_{i}$ given by Eq. (2) is the probability of $i$ packet arrivals during a frame [5] [7],

$$
P_{i}= \begin{cases}\left(\begin{array}{c}
N \\
i
\end{array}\right) \sigma^{i}(1-\sigma)^{N-i} & 0 \leq i \leq N \\
0 & i>N\end{cases}
$$

It can be proved that the Markov chain is irreducible, aperiodic and homogeneous, and thus it has unique stationary distribution $\pi_{T}=\left(\pi_{0_{T}}, \pi_{1_{T}}, \ldots, \pi_{L_{T}}\right)$ [11], where $\pi_{i_{T}}$ is the steady state probabilities of state $U_{i}$. Since each terminal transmits a packet in each frame except when its buffer is empty, the throughput of a TDMA system, $S_{T D M A}$, is

$$
S_{T D M A}=P_{s}(1)\left(1-(1-\sigma)^{N} \pi_{0_{T}}\right) .
$$

For the Markov chain of the C-TDMA system, its one step transition matrix $\left\{B_{C}\right\}_{i, j}$ is given by Eq. (4). In $\left\{B_{C}\right\}_{i, j}$, $\bar{P}_{H E}$ is the average transmission error probability of the headof-line (HOL) [12] in the FIFS buffer. Since the HOL in FIFS buffer could be for its first transmission or retransmitted by

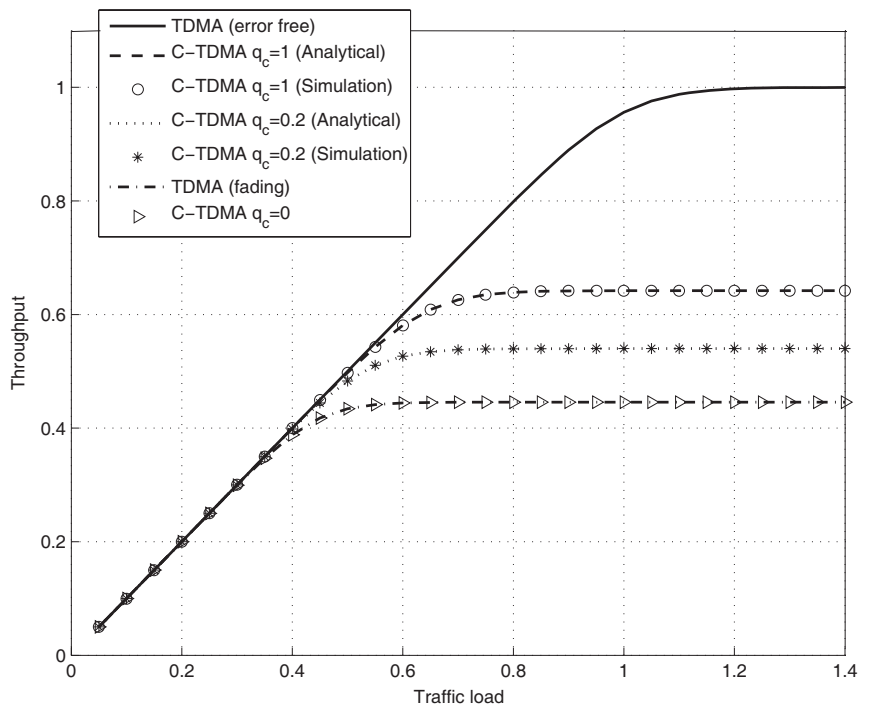

Fig. 1. Throughout of error-free TDMA, TDMA and C-TDMA in Rayleigh fading channels $(\mathrm{N}=10, \mathrm{~L}=10, \mathrm{SNR}=25 \mathrm{~dB})$.

the $n^{\text {th }}$ time due to previous failures, the calculation of $\bar{P}_{H E}$ is determined by the probability of the HOL transmitted or retransmitted by the $n^{\text {th }}$ time and the packet error rate with $k-1$ cooperative terminals. We use $P_{n^{t h}}$ to represent the probability of the HOL transmitted for the first time $(n=0)$ or retransmitted by the $n^{\text {th }}$ time $(n=1,2, \ldots)$. Moreover, let $Q_{r}(t)$ denote the probability that $t$ terminals correctly receive the source packet, $Q_{r}(t)=\left(\begin{array}{c}N-1 \\ t\end{array}\right)\left[P_{s}(1)\right]^{t}\left[P_{e}(1)\right]^{N-1-t}$, and $Q_{c}(t, k)$ donate the probability that $k$ out of $t$ terminals choose to involve in cooperation, $Q_{c}(t, k)=\left(\begin{array}{l}t \\ k\end{array}\right) q_{c}^{k}\left(1-q_{c}\right)^{t-k}$. Therefore, the probability of the HOL in the FIFS buffer being transmitted/retransmitted by the $n^{\text {th }}$ time, $P_{n^{t h}}$, can be represented by

$$
\left\{\begin{array}{lr}
P_{0^{t h}}, & n=0 \\
P_{0^{t h}} P_{e}(1), & n=1 \\
P_{0^{t h}} P_{e}(1)\left\{\sum_{t=1}^{N-1} Q_{r}(t) \sum_{k=1}^{t} Q_{c}(t, k)\left[P_{e}(k+1)\right]^{n-1}+(1-\right. \\
\left.\left.\sum_{t=1}^{N-1} Q_{r}(t) \sum_{k=1}^{t} Q_{c}(t, k)\right)\left[P_{e}(1)\right]^{n-1}\right\}, & n \geq 2
\end{array}\right.
$$

Since $\sum_{n=0}^{\infty} P_{n^{t h}}=1$, the specific value of $P_{0^{t h}}$ can be calculated by Eq. (6) and thus each specific value of $P_{n^{t h}}$ can be calculated by Eq. (5). Finally, $\bar{P}_{H E}$ can be calculated by Eq. (7) and the average correct transmission probability of the HOL in the FIFS buffer, $\bar{P}_{H S}$, is calculated using $\bar{P}_{H S}=$ $1-\bar{P}_{H E}$. The Markov chain of the C-TDMA system also has unique stationary distributions $\pi_{C}=\left(\pi_{0_{C}}, \pi_{1_{C}}, \ldots, \pi_{L_{C}}\right)$ [11], where $\pi_{i_{C}}$ is the steady state probabilities of state $U_{i}$. The throughput of a C-TDMA system, $S_{C-T D M A}$, is thus

$$
S_{C-T D M A}=\bar{P}_{H S}\left(1-(1-\sigma)^{N} \pi_{0_{C}}\right)
$$

\section{Analytical and Simulation Results}

In this section, we compare the throughput of TDMA with C-TDMA systems in Rayleigh fading channels. The performance of a TDMA system in an error-free channel is also illustrated in Fig. 1 based on the results in [7]. In both our simulation and analytical analysis evaluations, SNR is assumed to be $25 \mathrm{~dB}$, the packet length is 1024 bits, and the fading is assumed to be constant within each time 


$$
\begin{aligned}
& \mathbf{B}_{\mathbf{T}}=\left(\begin{array}{ccccc}
P_{0}+P_{1} P_{s}(1) & P_{1} P_{e}(1)+P_{2} P_{s}(1) & P_{2} P_{e}(1)+P_{3} P_{s}(1) & \ldots & 1-P_{0}-\sum_{i=1}^{L} P_{i} P_{e}(1)-\sum_{i=1}^{L-1} P_{i} P_{e}(1) \\
P_{0} P_{s}(1) & P_{0} P_{e}(1)+P_{1} P_{s}(1) & P_{1} P_{e}(1)+P_{2} P_{s}(1) & \ldots & 1-\sum_{i=0}^{L-1} P_{i} P_{s}(1)-\sum_{i=0}^{L-2} P_{i} P_{e}(1) \\
0 & P_{0} P_{s}(1) & P_{0} P_{e}(1)+P_{1} P_{s}(1) & \ldots & 1-\sum_{i=0}^{L-2} P_{i} P_{s}(1)-\sum_{i=0}^{L-3} P_{i} P_{e}(1) \\
\ldots & \ldots & \ldots & \ldots & \ldots \\
0 & 0 & 0 & P_{0} P_{s}(1) & 1-P_{0} P_{s}(1)
\end{array}\right) \\
& \mathbf{B}_{\mathbf{C}}=\left(\begin{array}{ccccc}
P_{0}+P_{1} \bar{P}_{H S} & P_{1} \bar{P}_{H E}+P_{2} \bar{P}_{H S} & P_{2} \bar{P}_{H E}+P_{3} \bar{P}_{H S} & \ldots & 1-P_{0}-\sum_{i=1}^{L} P_{i} \bar{P}_{H S}-\sum_{i=1}^{L-1} P_{i} \bar{P}_{H E} \\
P_{0} \bar{P}_{H S} & P_{0} \bar{P}_{H E}+P_{1} \bar{P}_{H S} & P_{1} \bar{P}_{H E}+P_{2} \bar{P}_{H S} & \ldots & 1-\sum_{i=0}^{L-1} P_{i} \bar{P}_{H S}-\sum_{i=0}^{L-2} P_{i} \bar{P}_{H E} \\
0 & P_{0} \bar{P}_{H S} & P_{0} \bar{P}_{H E}+P_{1} \bar{P}_{H S} & \ldots & 1-\sum_{i=0}^{L-2} P_{i} \bar{P}_{H S}-\sum_{i=0}^{L-3} P_{i} \bar{P}_{H E} \\
\ldots & \ldots & \ldots & \ldots & \ldots \\
0 & 0 & 0 & P_{0} \bar{P}_{H S} & 1-P_{0} \bar{P}_{H S}
\end{array}\right) \\
& P_{0^{t h}}=\frac{1}{1+P_{e}(1)+P_{e}(1)\left\{\sum_{t=1}^{N-1} Q_{r}(t) \sum_{k=1}^{t} Q_{c}(t, k) \frac{P_{e}(k+1)}{1-P_{e}(k+1)}+\left(1-\sum_{t=1}^{N-1} Q_{r}(t) \sum_{k=1}^{t} Q_{c}(t, k)\right) \frac{P_{e}(1)}{1-P_{e}(1)}\right\}} \\
& \bar{P}_{H E}=\sum_{n=0}^{\infty} P_{n^{t h}} \operatorname{Pr}\left(\text { Error } \mid n^{t h}\right) \\
& =P_{0^{t h}} P_{e}(1)\left\{\sum_{t=1}^{N-1} Q_{r}(t) \sum_{k=1}^{t} Q_{c}(t, k) \frac{P_{e}(k+1)}{1-P_{e}(k+1)}+\left(1-\sum_{t=1}^{N-1} Q_{r}(t) \sum_{k=1}^{t} Q_{c}(t, k)\right) \frac{P_{e}(1)}{1-P_{e}(1)}\right\}
\end{aligned}
$$

slot (block fading). The theoretical and simulation results of C-TDMA systems are illustrated in Fig. 1 for different $q_{c}$ values. Note that simulation results are obtained using Matlab and the simulation accuracy is maintained based on the convergence of the simulation results. It is also noted that the analytical and simulation results match very well. In Fig. 1, the maximum throughput of a TDMA system in an error-free channel is 1 under a high traffic load. However, the maximum throughput of TDMA is only about 0.45 without using cooperative diversity in a Rayleigh fading channel. For a C-TDMA system, when $q_{c}$ value increases from 0.2 to 1 , there are more terminals in the cooperation terminal set. When $q_{c}$ is 0 , there is no cooperative transmission and the system becomes a TDMA system without cooperative diversity. It is seen that the maximum throughput of the C-TDMA system has $44 \%$ increase compared to the TDMA system due to the cooperation transmissions when $q_{c}$ equals to 1 .

\section{Conclusions}

In this paper, we introduced cooperative transmissions into the conventional TDMA system and obtained a cooperative TDMA protocol. Based on the cooperation transmissions from both a source terminal and other neighbor terminals in a wireless network, a diversity gain is obtained to improve the probability of correct packet reception, resulting in a $44 \%$ throughput increase as compared with conventional TDMA.

\section{REFERENCES}

[1] F. H. P. Fitzek and M. D. Katz, Cooperation in Wireless Networks: Principles and Applications, 1st edition. Dordrecht, The Netherlands: Springer, 2006.

[2] P. Liu, Z. Tao, S. Narayanan, T. Korakis, and S. S. Panwar, "CoopMAC: a cooperative MAC for wireless LANs," IEEE J. Sel. Areas Commun., vol. 25, no. 2, pp. 340-354, Feb. 2007.

[3] M. S. Gokturk and O. Gurbuz, "Cooperation in wireless sensor networks: design and performance analysis of a MAC protocol," in Proc. IEEE ICC'08.

[4] M. S. Gokturk, O. Ercetin, and O. Gurbuz, "Throughput analysis of ALOHA with cooperative diversity," IEEE Commun. Lett., vol. 12, no. 6, pp. 468-470, June 2008.

[5] A. K. Sadek, K. J. R. Liu, and A. Ephremides, "Collaborative multipleaccess protocols for wireless networks," in Proc. IEEE ICC'06, vol. 10, pp. 4495-4500, June 2006.

[6] M. Dohler, E. Lefranc, and H. Aghvami, "Space-time block codes for Virtual antenna arrays," in PIMRC, Lisbon, Portugal, Sep. 2002.

[7] D. Zheng and Y. D. Yao, "Throughput performance evaluation of two-tier TDMA for sensor networks," IEEE Sarnoff Symposium, Princeton, NJ, 2009.

[8] S. M. Alamouti, "A simple transmit diversity technique for wireless communications," IEEE J. Sel. Areas Commun., vol. 16, no. 8, pp. 14511458, Oct. 1998

[9] A. J. Goldsmith, Wireless Communications, 1st edition. New York: Cambridge University Press, 2005.

[10] N. M. Dor, "Guide to the length of buffer storage required for random (Poisson) input and constant output rates," IEEE Trans. Electron. Comput., vol. 16, no. 5, pp. 683-684, Oct. 1967.

[11] W. J. Stewart, Introduction to the Numerical Solution of Markov Chains, 1st edition. Princeton, NJ: Princeton University Press, 1994.

[12] A. Matthew, K. Krishnan, R. Kavita, S. Alexander, and W. Phil, "Providing quality of service over a shared wireless link," IEEE Commun. Mag., vol. 39, no. 2, pp. 150-154, Feb. 2001. 